\title{
El paciente que padece un trastorno de personalidad en el trabajo
}

\section{Patients who suffer from a personality disorder at work}

\section{Macarena Gálvez Herrer}

UVOPSE. Unidad de Valoración y Orientación al profesional sanitario enfermo. Comunidad de Madrid. Madrid. España.

\section{José Carlos Mingote Adán}

Facultad de Psicología de la Universidad Autónoma de Madrid.

Madrid. España.

\section{Bernardo Moreno Jiménez}

Facultad de Psicología de la Universidad Autónoma de Madrid.

Madrid. España.

Recibido: 20-09-10

Aceptado: 29-09-10

\section{Correspondencia:}

Macarena Gálvez Herrer

Pabellón 8. Ciudad Universidad

28040 Madrid. España.

Tfno: 913303926

e-mail: mgalvez.hcsc@salud.madrid.org

\section{Resumen}

En el lugar de trabajo se hacen patentes las relaciones interpersonales entre los trabajadores, se expresan las subjetividades de cada individuo, se toman decisiones influenciadas por estilos personales, estilos de liderazgo, etc. Todo en el trabajo es "personal".

Los individuos con trastornos de personalidad son altos consumidores de asistencia médica por sintomatología sin diagnóstico claro, el trastorno converge en numerosas ocasiones con otros tipos de alteraciones psiquiátricas y media en complicaciones sociales. Tanto para la medicina general, psiquiátrica, como laboral, se trata en muchas ocasiones de personas de difícil manejo.

Siguiendo una línea de trabajo en la que analizamos la interacción entre salud mental y trabajo, el objetivo del presente artículo es realizar una exposición de los diferentes trastornos de la personalidad revisando sus características, identificación y criterios diagnósticos, analizando su posible expresión en el lugar de trabajo y señalando alguna de las principales líneas de afrontamiento y manejo, tanto laboral como clínico.

Med Segur Trab (Internet) 2010; 56 (220): 226-247

Palabras clave: Trastorno de personalidad, trabajo, riesgos laborales, conflicto interpersonal. 


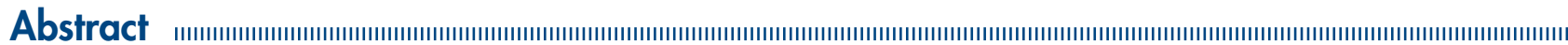

In the working place the relationships between workers are shown, each individual's subjectivities are expressed, and the decisions made are influenced by personal styles, leadership styles, etc. Everything at work is "personal."

Individuals with personality disorders are high consumers of medical care due to symptoms with no clear diagnosis, the disorder on numerous occasions converge with another types of psychiatric alterations and it is involved in social complications. For general, psychiatric and labour medicine, most of the times, these are people difficult to manage.

Following a line of work in which we analyzed the interaction between mental health and work, the objective of this document is to present the different personality disorders reviewing their characteristics, identification and diagnostic criteria, analyzing their possible expression in the working place, and pointing out some of the main lines for their assumption and management, both from a labour and clinic standpoint.

Med Segur Trab (Internet) 2010; 56 (220): 226-247

Key words: Personality disease, work, labour risk, interpersonal conflict. 


\section{INTRODUCCIÓN}

En el lugar de trabajo se hacen patentes las relaciones interpersonales entre los trabajadores, se expresan las subjetividades de cada individuo, se toman decisiones influenciadas por estilos personales, estilos de liderazgo, etc. Todo en el trabajo es "personal".

Una primera aproximación al concepto de personalidad podría presentarla como la configuración que explica las diferentes conductas ante un mismo medio. Sin embargo, la personalidad no es sólo lo que diferencia, sino también el principio de organización de la propia conducta, la organización interna de la experiencia y la acción individual. Además de ser reactiva, es pro-activa. Se caracteriza por su capacidad para organizar la conducta sin atender a estímulos físicamente presentes, atendiendo a objetivos, metas y planes que el individuo se plantea obtener (incluidos los laborales). Esta organización de la personalidad se construye en función de cinco variables básicas: observables (conducta expresa), no observables (mundo interno, pensamientos, emociones, imágenes, intenciones), biológicas (factores genéticos, neurológicos, neuroendocrinos, etc.), históricas (biográficas) y sociodemográficas (como sexo, edad, cultura, etc.) ${ }^{1}$.

Académicamente, el estudio de la personalidad se ha centrado en el análisis de la estructura de la misma, privilegiando el estudio de la consistencia sobre el cambio y la taxonomía de sus componentes. De forma complementaria, es posible hablar de una psicología de la personalidad centrada en los procesos, más cerca de una concepción de la personalidad como sujeto psicológico, y no sólo como estructura de rasgos. Desde esta perspectiva hablaremos de el sujeto cognitivo, la personalidad emocional, las conductas de la persona, la construcción social de la personalidad y los procesos autorreferenciales (autorregulación e identidad personal).

Cuando existe un trastorno de personalidad, se producen un conjunto de perturbaciones que afecta a esas dimensiones emocionales, afectivas, motivacionales y de relación social de los individuos. Cuando ese trastorno de personalidad se hace patente en el lugar de trabajo se ve afectada la calidad del mismo así como la salud individual y colectiva de los trabajadores. Según Millon y Everly ${ }^{2}$, una evaluación multidimensional incluiría los aspectos relativos a conducta aparente, interpersonal, estilo cognitivo, expresión afectiva, percepción de sí mismo, mecanismo de defensa utilizado y diagnóstico diferencial respecto a otros trastornos de la personalidad. Características comunes a las personas con trastorno de personalidad son:

1. Disminución de la capacidad de introspección (insight) y de contacto con las propias emociones.

2. Dificultad para desarrollar relaciones interpersonales empáticas.

3. Alteraciones del comportamiento. Como pueden ser la impulsividad (paso al acto), conductas agresivas, alteración del humor, etc., según el trastorno de personalidad.

Los individuos con trastornos de personalidad son altos consumidores de asistencia médica por sintomatología sin diagnóstico claro, el trastorno converge en numerosas ocasiones con otros tipos de alteraciones psiquiátricas y media en complicaciones sociales. Tanto para la medicina general, psiquiátrica, como laboral, se trata en muchas ocasiones de personas de difícil manejo. Es comúnmente aceptada una prevalencia entorno al $10-15 \%$ de la población adulta, de los que casi la mitad corresponden a trastornos graves (antisocial, límite, narcisista, paranoide y esquizoide). Por edades, los trastornos del grupo B son más frecuentes en jóvenes, mientras que los grupos A y C se distribuyen de forma más equilibrada. Respecto al género, los trastornos de personalidad narcisista y antisocial son más frecuentes en hombres y los de tipo límite e histriónico en mujeres.

Los trastornos de personalidad son difíciles de diagnosticar y más aún de explicar a los pacientes en tratamiento, porque supone una "etiqueta" que puede vivirse como especialmente problemática, descalificativa y de probable mal pronóstico. Será un reto explicar a la persona afectada que la esencia de su problema es tener "dificultades 
persistentes en las relaciones interpersonales", ayudarle a elegir una posición activa y reflexiva, y a afrontar los aspectos perturbadores de su personalidad ante si mismo y ante los demás, comprendiendo cómo algunos de sus rasgos de personalidad, dentro de ciertos límites, pueden ser más adaptativos o funcionales.

Siguiendo una línea de trabajo en la que analizamos la interacción entre salud mental y trabajo ${ }^{3,4}$, el objetivo del presente artículo es realizar una exposición de los diferentes trastornos de la personalidad revisando sus características, identificación y criterios diagnósticos (según DSM-IV) ${ }^{5}$, analizando su posible expresión en el lugar de trabajo y señalando alguna de las principales líneas de afrontamiento y manejo, tanto laboral como clínico. Mantenemos esa clasificación por estar actualmente vigente, si bien es necesario señalar que está en proceso de revisión y se prevee la aparición del DSM-V para el 2013. En dicha revisión, está prevista una reestructuración de la categorización de los trastornos de personalidad, así como la evaluación de su severidad en función de aspectos de adaptación y funcionamiento entorno a si mismo y a las relaciones interpersonales (para ampliar esta información se puede consultar el primer borrador del DSM-V en la página web oficial)

\section{GRUPO A (Raros o excéntricos)}

Este grupo de trastornos se caracteriza por un patrón penetrante de cognición (por ejemplo de sospecha), expresión (como un lenguaje extraño) y relación con otros (con conductas de aislamiento por ejemplo) anormales. Incluye al trastorno paranoide de la personalidad, esquizoide y esquizotípico.

El trastorno PARANOIDE de personalidad se caracteriza por una desconfianza y suspicacia general desde el inicio de la edad adulta, de forma que las intenciones de los demás son interpretadas como maliciosas en diversos contextos, como lo indican cuatro (o más) de los siguientes puntos:

1. Sospecha, sin base suficiente, que los demás se van a aprovechar de ellos, les van a hacer daño o les van a engañar

2. Preocupación por dudas no justificadas acerca de la lealtad o la fidelidad de los amigos y socios

3. Reticencia a confiar en los demás por temor injustificado a que la información que compartan vaya a ser utilizada en su contra

4. En las observaciones o los hechos más inocentes, vislumbra significados ocultos que son degradantes o amenazadores

5. Alberga rencores durante mucho tiempo, por ejemplo, no olvida los insultos, injurias o desprecios

6. Percibe ataques a su persona o a su reputación que no son aparentes para los demás y está predispuesto a reaccionar con ira o a contraatacar

7. Sospecha repetida e injustificadamente que su cónyuge o su pareja le es infiel

Cuando hablamos de un trastorno ESQUIZOIDE, nos referimos a un patrón general de distanciamiento de las relaciones sociales y de restricción de la expresión emocional en el plano interpersonal, que comienza al principio de la edad adulta y que se da en diversos contextos, como lo indican cuatro (o más) de los siguientes puntos:

1. Ni desea ni disfruta de las relaciones personales, incluido el formar parte de una familia

2. Escoge casi siempre actividades solitarias

3. Tiene escaso o ningún interés en tener experiencias sexuales con otra persona

4. Disfruta con pocas o ninguna actividad

5. No tiene amigos íntimos o personas de confianza, aparte de los familiares de primer grado 
6. Se muestra indiferente a los halagos o las críticas de los demás

7. Muestra frialdad emocional, distanciamiento o aplanamiento de la afectividad

El trastorno ESQUIZOTIPICO de personalidad se expresa mediante un patrón general de déficit sociales e interpersonales asociados a malestar agudo y una capacidad reducida para las relaciones personales, así como distorsiones cognoscitivas o perceptivas y excentricidades del comportamiento, que comienzan al principio de la edad adulta y se dan en diversos contextos, como lo indican cinco (o más) de los siguientes puntos:

1. Ideas de referencia (excluidas las ideas delirantes de referencia)

2. Creencias raras o pensamiento mágico que influye en el comportamiento y no es consistente con las normas subculturales (superstición, creer en la clarividencia, telepatía o "sexto sentido"; en niños y adolescentes, fantasías o preocupaciones extrañas)

3. Experiencias perceptivas inhabituales, incluidas las ilusiones corporales

4. Pensamiento y lenguaje raros (vago, circunstancial, metafórico, sobre-elaborado o estereotipado)

5. Suspicacia o ideación paranoide

6. Afectividad inapropiada o restringida

7. Comportamiento o apariencia raros, excéntricos o peculiares

8. Falta de amigos íntimos o desconfianza aparte de los familiares de primer grado

9. Ansiedad social excesiva que no disminuye con la familiarización y que tiende a asociarse con temores paranoides más que con juicios negativos sobre uno mismo

\section{a) Características y manejo en el lugar de trabajo (Grupo A)}

Los trastornos del grupo A son quizás algunos de los de mayor dificultad de manejo en el lugar de trabajo incluso, en ocasiones, su gravedad impide una correcta inserción laboral. La expresividad de su sintomatología, sin embargo, suele permitir su reconocimiento, detección y diagnóstico.

En el caso de la personalidad paranoica, las relaciones interpersonales son especialmente difíciles debido a que intentos de contacto y acercamiento pueden ser interpretados como hostiles o persecutorios. El trabajador con personalidad paranoide pude ser muy discutidor, callado o de frecuentes quejas, presentando hostilidad hacia los otros. Son especialmente sensibles a la crítica y con gran necesidad de autonomía ${ }^{6}$ por lo que pueden proyectar hacia los demás, fines y motivos indeseables propios.

En algunos casos de percepción de acoso laboral, al analizar la sintomatología y rasgos de personalidad asociados en la posible víctima, no es infrecuente encontrar indicadores de paranoia, siendo importante discernir mediante un adecuado diagnóstico diferencial, si se trata de sintomatología reactiva al riesgo psicosocial vivido o predecesora de su percepción subjetiva.

En una interacción funcional con un trabajador con personalidad paranoica, la relación será más fácil si se procura que sea limitada a "lo profesional" y emocionalmente no intima, con estilos comunicativos centrados en datos objetivos sobre el trabajo y la tarea a desempeñar, sin obligarles a expresar sus emociones y entendiendo que no lo harán a menudo.

Tabla 1. Trastorno Paranoide en el Trabajo:

Conducta aparente: Impresiona de vigilante, desconfiado.

Conducta interpersonal: Frecuentes quejas, hipersensible a la crítica (de compañeros y supervisor).

Estilo cognitivo: Suspicaz, conspirador (en las relaciones laborales interpersonales).

Percepción de sí mismo: Incapacidad de percibir los propios errores (son los demás)

Mecanismo de defensa: Proyección 
En el caso de la personalidad esquizoide, el desapego hará que los trabajadores con este trastorno prefieran puestos de trabajo aislados, sin tareas de trabajo en equipo y con escasa interacción personal. Serán más efectivos en puestos donde no sean requisitos de éxito la empatía o la expresión de afectos. Como señalaron Beck y Freeman (1990, p.125) "se consideran como observadores en lugar de participantes en el mundo que les rodea" y por ello, la formación y entrenamiento en habilidades sociales será un elemento clave de integración sociolaboral.

Son mínimamente introspectivos, por ello se puede observar una participación muy superficial con el grupo de trabajo, en la conversación dentro del mismo, etc. Pueden tender a analizar las experiencias más emocionales desde una perspectiva impersonal, casi mecánica, empleando la intelectualización como mecanismo de defensa. Es decir, describiendo dichas experiencias afectivas e interpersonales en términos de hechos reales, prestando solo atención a los aspectos formales de los acontecimientos sociales para no involucrarse en ellos.

Tabla 2. Trastorno Esquizoide en el Trabajo:

Conducta aparente: Apática, baja de energía, perezosa en el trabajo, reservada.

Conducta interpersonal: Distanciamiento de las relaciones sociales, aislamiento social del grupo de trabajo,

restricción de la expresión emocional.

Estilo cognitivo: Empobrecido, mínimamente introspectivo.

Percepción de sí mismo: Pueden variar desde complacientes a faltos de vitalidad en casos extremos.

Mecanismo de defensa: Intelectualización

Esas conductas de aislamiento social también se dan en el trastorno esquizotipico de la personalidad, pero en este caso, el trabajador además se comporta de manera extraña, de forma desconfiada y suele tener creencias extravagantes e ideas de referencia, pensando que sucesos insignificantes de la actividad laboral o el lugar de trabajo se relacionan de manera directa con ellos. Esta situación puede ser foco de conflictos interpersonales.

Su evaluación cognitiva demuestra una disminución de la capacidad de ejecución en pruebas de memoria y aprendizaje, lo que ha llevado a algunos autores a hipotetizar sobre algún tipo de daño en el hemisferio izquierdo ${ }^{8}$ y puede relacionarse con posibles dificultades de rendimiento. El estilo cognitivo puede variar desde rumiador y autístico (en casos menos graves) a desorientados y con pensamiento no lógico, perdiéndose en aspectos irrelevantes de la tarea.

Se consideran a sí mismos como desamparados y tienen gran dificultad para encontrar un sentido al trabajo y a la vida en general. En casos graves, puede aparecer la anulación como mecanismo de defensa o auto-purificación para intentar arrepentirse de alguna conducta indeseable o móvil no deseado. Puede tomar forma de rituales (como el lavado de manos) o actos "mágicos".

Tabla 3. Trastorno Esquizotipico en el Trabajo:

Conducta aparente: Excéntrica o extravagante

Conducta interpersonal: Aislamiento social, ansiedad ante desafíos sociales.

Estilo cognitivo: Desde rumiador a desconcertado.

Percepción de sí mismo: Desamparado, con falta de sentido en el trabajo y en la vida.

Mecanismo de defensa: Anulación.

\section{b) Abordaje psiquiátrico y psicológico (Grupo A)}

Quienes sufren un trastorno de personalidad del grupo A, desconfían de la mayoría de la gente y tienen gran dificultad en la expresión y manejo de las emociones, por ello es poco probable que busquen ayuda cuando la necesitan, y si lo hacen no será fácil desarrollar una relación de confianza, necesaria para que la psicoterapia sea efectiva. Con frecuencia, cuando buscan ayuda es porque otro activador interfiere en su vida como la ansiedad, la depresión, los conflictos interpersonales en el trabajo o la pérdida del mismo. 
La relación con el terapeuta será clave para crear un espacio de relación saludable, conexión social y análisis de del papel funcional o disfuncional del paciente en su entorno.

Cuestionar directamente las sospechas del trabajador paranoico suele ser bastante ineficaz, será necesario intentar de forma prioritaria el mejor vínculo terapéutico posible. Para ello, comenzar por las propias metas del paciente y su sensación de control y capacidad sobre las mismas, generará de forma progresiva, una mayor confianza y permitirá abordar las interacciones disfuncionales, los aspectos emocionales y la comprensión del mundo y de los demás propias del trastorno.

En este grupo de trastornos de la personalidad, desde la psicoterapia serán objetivos básicos la reducción de la sensibilidad ante las críticas, el aislamiento social y el entrenamiento en habilidades sociales (atención social, procesamiento de la información, emisión de respuestas, comunicación no verbal y retroalimentación positiva y negativa).

Respecto al tratamiento farmacológico en el caso del trastorno paranoide de la personalidad, en la práctica clínica se utilizan antipsicóticos, siendo preferible emplear dosis bajas (olanzapina o risperidona). Cuando existe sintomatología obsesiva, disforia, ansiedad social y/o irritabilidad, se utilizan antidepresivos, especialmente serotoninérgicos o clomipramina. En el caso del trastorno esquizotípico, también se utilizan antipsicóticos a dosis bajas, especialmente ante ideas de referencia, alteraciones del pensamiento o paranoia.

\section{c) Caso clínico: Consulta por percepción de acoso laboral en un trabajador con trastorno de personalidad paranoide}

Hombre de 60 años, soltero, origen latinoamericano (con más de 20 años de residencia en España), médico de familia. Consulta por conflictos interpersonales en el lugar de trabajo, y percepción de acoso laboral. Niega existencia de antecedentes psiquiátricos. Historia laboral extensa como médico y suplente en diferentes Comunidades Autónomas. En la actualidad, estudiando Derecho, según expresa "para defenderme a mi mismo de este complot".

Tras la evaluación mediante entrevistas clínicas y cuestionarios de personalidad y sintomatología, se observan rasgos de personalidad obsesiva y rasgos narcisistas, minimización de problemas emocionales, temor a ser considerado enfermo mental, ausencia de conciencia de enfermedad. Ideaciones referenciales y de perjuicio irreductibles (personales y profesionales) de más de 20 años de evolución (en su ciudad natal, en el extranjero donde cursó estudios de postgrado y en España) por parte de diferentes personas e instituciones:

- Suplantación de identidad por parte de un familiar

- Difamación e información a la policía por un compañero de estudios

- Investigación, informes policiales falsos, dudas sobre su identidad sexual y presiones oficiales para que no la den trabajo o le echen de ellos, por parte de la policía (nacional e internacional).

- Acoso laboral percibido por parte de los compañeros actuales de trabajo: cambios en informes clínicos, percepción de crítica personal, conflictos interpersonales, conductas de aislamiento...

La intervención sobre el caso requirió la colaboración entre Servicio de Prevención de Riesgos Laborales, Centro de Salud Mental, de Atención Primaria y Colegio Profesional, con el fin de proceder a una baja laboral primero y a un proceso de invalidez posteriormente que considerara, de forma prioritaria, la correcta atención a la propia salud del facultativo, así como el posible riesgo a terceros de su trabajo asistencial. 


\section{GRUPO B (Emotivos y dramáticos)}

Estos trastornos se caracterizan, en ocasiones, por un patrón penetrante de violación de las normas sociales, comportamiento impulsivo, emotividad excesiva y grandiosidad. Presentan con frecuencia acting-out (exteriorización de sus rasgos), que puede conducir a expresivos enfados, comportamiento auto-abusivo y arranques de rabia. Incluye el trastorno histriónico, narcisista, antisocial y límite de la personalidad.

El trastorno HISTRIÓNICO de personalidad se da en un 2-3\% de la población general. Se trata de personas con un patrón general de excesiva emotividad y una búsqueda de atención, que suele observarse al principio de la edad adulta y que se da en diversos contextos, como lo indican cinco (o más) de los siguientes ítems:

1. No se siente cómodo en las situaciones en las que no es el centro de la atención.

2. La interacción con los demás suele estar caracterizada por un comportamiento sexualmente seductor o provocador.

3. Muestra una expresión emocional superficial y rápidamente cambiante.

4. Utiliza permanentemente el aspecto físico para llamar la atención sobre sí mismo.

5. Tiene una forma de hablar excesivamente subjetiva y carente de matices.

6. Muestra autodramatización, teatralidad y exagerada expresión emocional.

7. Es sugestionable, por ejemplo, fácilmente influenciable por los demás o por las circunstancias.

8. Considera sus relaciones más íntimas de lo que son en realidad.

Respecto al trastorno NARCISISTA, se expresa con un patrón general de grandiosidad (en la imaginación o en el comportamiento), una necesidad de admiración y una falta de empatía, que empiezan al principio de la edad adulta y que se dan en diversos contextos como lo indican cinco (o más) de los siguientes ítems:

1. Tiene un grandioso sentido de autoimportancia (por ejemplo exagera los logros, capacidades, espera ser reconocido como superior sin unos logros proporcionados).

2. Está preocupado por fantasías de éxito ilimitado, poder, brillantez, belleza y amor imaginarios.

3. Cree que es "especial" y único y que sólo puede ser comprendido por, o sólo puede relacionarse con otras personas (o instituciones) que son especiales de alto status.

4. Exige una admiración excesiva.

5. Es muy pretencioso, por ejemplo tiene expectativas irrazonables de recibir un trato de favor especial o de que se cumplan automáticamente sus expectativas.

6. Es interpersonalmente explotador, por ejemplo saca provecho de los demás para alcanzar sus propias metas.

7. Carece de empatía: es reacio a reconocer o identificarse con los sentimientos y necesidades de los demás.

8. Frecuentemente envidia a los demás y cree que los demás lo envidian a él.

9. Presenta comportamientos o actitudes arrogantes o soberbias.

El trastorno ANTISOCIAL supone el desprecio y violación de los derechos de los demás. Se presenta desde la edad de 15 años, como lo indican tres (o más) de los siguientes ítems:

1. Fracaso para adaptarse a las normas sociales en lo que respecta al comportamiento legal, como lo indica el perpetrar repetidamente actos que son motivo de detención

2. Deshonestidad, indicada por mentir repetidamente, utilizar un alias, estafar a otros para obtener un beneficio personal o por placer

3. Impulsividad o incapacidad para planificar el futuro 
4. Irritabilidad y agresividad, indicados por peleas físicas repetidas o agresiones

5. Despreocupación imprudente por su seguridad o la de los demás

6. Irresponsabilidad persistente, indicada por la incapacidad de mantener un trabajo con constancia o de hacerse cargo de obligaciones económicas

7. Falta de remordimientos, como lo indica la indiferencia o la justificación del haber dañado, maltratado o robado a otros

La personalidad borderline o con trastorno LÍMITE incluye un patrón general de inestabilidad en las relaciones interpersonales, la autoimagen y la efectividad, y una notable impulsividad, que comienzan al principio de la edad adulta y se da en diversos contextos, como lo indican cinco (o más) de los siguientes ítems:

1. Esfuerzos frenéticos para evitar un abandono real o imaginado.

2. Patrón de relaciones interpersonales inestables e intensas caracterizado por la alternancia entre los extremos de idealización y devaluación.

3. Alteración de la identidad: autoimagen o sentido de sí mismo acusada y persistentemente inestable.

4. Impulsividad en al menos dos áreas, que es potencialmente dañina para sí mismo (gastos, sexo, abuso de sustancias, conducción temeraria, trastornos de la alimentación).

5. Comportamientos, intentos o amenazas suicidas recurrentes, o comportamiento de automutilación.

6. Inestabilidad afectiva debida a una notable reactividad del estado de ánimo (episodios de intensa disforia, irritabilidad o ansiedad, que suelen durar unas horas y rara vez unos días)

7. Sentimientos crónicos de vacío.

8. Ira inapropiada e intensa o dificultades para controlar la ira (muestras frecuentes de mal genio, enfado constante, peleas físicas recurrentes).

9. Ideación paranoide transitoria relacionada con el estrés o síntomas disociativos graves

\section{a) Características y manejo en el lugar de trabajo (Grupo B)}

En el caso del trastorno de personalidad histriónico, sus actitudes pueden ocasionar, en no pocas ocasiones, dificultades de interacción con el equipo de trabajo, ya que la teatralidad de su conducta puede convertir hechos triviales de la cotidianeidad laboral (positivos o conflictivos) en acontecimientos de gran relevancia, ofreciendo una respuesta emocional distorsionada ante los mismos. Además, los conflictos pueden devenir de un estilo cognitivo asociado impresionista ${ }^{9}$, es decir, que se caracterizará por una tendencia a ver las situaciones laborales de manera muy general, en términos dicotómicos de blanco o negro (o estás conmigo o estás contra mí, por ejemplo).

Oldham y Morris ${ }^{10}$ recomiendan no reprimir a estas personas sus ideas por hacer cosas nuevas, sus planes y su capacidad para disfrutar de las experiencias, necesitan que se les valore y elogie pero de forma sincera, y ante estallidos de ira, la mejor manera de sobrellevar la situación es dejar que amaine. Ellos evitarán profundizar, prestarán más atención a los detalles externos que al fondo de las cuestiones. Esta superficialidad conducirá a la disociación como principal mecanismo de defensa, separando su "identidad real" de su "identidad pública".

\section{Tabla 4. Trastorno Histriónico en el Trabajo:}

Conducta aparente: Afectada, teatral.

Conducta interpersonal: Sociables pero dependientes del refuerzo ajeno. Demandantes de atención, seductores.

Estilo cognitivo: Caprichoso, centrado en el detalle externo, no en el fondo.

Percepción de sí mismo: Sociable.

Mecanismo de defensa: Disociación. 
En el lugar de trabajo, el individuo narcisista requerirá continuamente el reconocimiento social y recompensas externas, necesitando la admiración del staff y compañeros, adjudicándose sin problemas parte de los éxitos, pero denegando culpabilidad en los fallos. Esta situación puede ocasionar especial dificultad en su supervisión y en la relación con los compañeros. La falta de empatía puede además ser causa de conflictividad interpersonal y situaciones de abuso emocional, infravalorando o devaluando las conductas de los demás, prestando atención a las obligaciones del otro sobre las propias y racionalizando los propios fallos o actos socialmente inaceptables ocurridos en el lugar de trabajo.

Tanto el trabajador con personalidad histriónica como el narcisista, no desean pasar inadvertidos, necesitan sentirse importantes y reafirmar su identidad y autoestima con demandas constantes de atención. Esta situación puede dificultarles el trabajo en solitario, donde la apatía y el aburrimiento pueden hacer mella, prefiriendo, frecuentemente, el trabajo con al menos un grupo de personas de los que recibir atención. Requerirán de un estilo de supervisión con frecuente retroalimentación o feedback, pero donde las habilidades asertivas del líder serán especialmente importantes al referirse a aspectos negativos del desempeño profesional con "diplomacia".

Tabla 5. Trastorno Narcisista en el Trabajo:

Conducta aparente: Arrogante, pomposo.

Conducta interpersonal: Manipulador, explotador.

Estilo cognitivo: Atención a las obligaciones del otro.

Percepción de sí mismo: Exageración de las propias habilidades.

Mecanismo de defensa: Racionalización.

Los conflictos en relación con el trastorno antisocial y límite de personalidad pueden ser de carácter más violento. Las personas con conductas violentas, según Marie France Hirigoyen ${ }^{11}$, suelen tener una serie de características propias de los trastornos de personalidad de tipo narcisista, paranoide o antisocial, tales como:

- El sujeto tiene una idea grandiosa de su propia importancia.

- Le absorben fantasías ilimitadas de éxito y de poder.

- Se considera especial y único.

- Tiene una necesidad excesiva de ser admirado.

- Piensa que se le debe todo.

- Explota al otro en sus relaciones interpersonales.

- Carecen de empatía aunque pueden ser muy brillantes socialmente.

- Puede fingir que entiende los sentimientos de los demás.

- Tiene actitudes y comportamientos arrogantes.

Estas actitudes (con sus componentes cognitivos, emocionales y conductuales) en el lugar de trabajo, pueden ser claramente desencadenantes o facilitadoras de conflictos interpersonales, situaciones de acoso, incremento del estrés psicosocial, mala distribución del trabajo, evitación de responsabilidades, etc. Es decir, desencadenantes o facilitadoras de condiciones de trabajo con riesgo psicosocial o incluso de la pérdida del mismo. Además, ambos trastornos de personalidad son los que aparecen con mayor comorbilidad en relación con consumo de sustancias y adicciones ${ }^{12}$.

A las personas con estilo antisocial de personalidad no les amedrentan las situaciones que asustan a la mayoría de las personas, el miedo es algo emocionante y por tanto pueden cometer actos temerarios en el lugar de trabajo, lo que puede ser causa de accidente laboral. Expresan abiertamente sus emociones, son ambiciosas, necesitan a su lado a personas que le den mucho a cambio de poco, y con frecuencia se saltan las normas, aunque pueden ser buenos trabajadores si la tarea les ofrece nuevos retos y estimulación novedosa de forma continua ${ }^{13}$. En los casos más leves pueden ser muy competitivos en el trabajo, en los más graves, la transgresión de las normas y la ley puede ser causa de la pérdida del empleo. 
Bajo un estrés persistente o especialmente agudo, pueden distorsionar la realidad para reducir la tensión y la ansiedad, reaccionando con una respuesta exagerada e impulsiva en la que muestran sus pensamientos, emociones y conducta abiertamente, aunque esto sea ofensivo socialmente (acting out). El objetivo será desahogar (mediante una fuerte discusión por ejemplo), la tensión interna.

En el DSM actual (DSM-IV-TR) se ha producido un desplazamiento hacia los aspectos más delincuenciales de la personalidad del psicópata, disminuyendo la importancia de la experiencia interna, de la constelación de valores y de la insensibilidad ante el otro, aunque no sean delictivas. Sin embargo, tanto desde la investigación como desde la clínica se insiste en la importancia de la psicopatía subclínica, de aquella que no origina una transgresión de la ley, en parte como consecuencia de un alto nivel de inteligencia y adaptación legal y oficial, aunque estén presentes elevados niveles de indiferencia al dolor ajeno, por omisión e incluso por acción.

En el ámbito de la investigación se ha desarrollado el modelo de la llamada "Triada oscura de la personalidad" aludiendo con ello a la presencia conjunta del maquiavelismo, el narcisismo y la psicopatía subclínica ${ }^{14}$. Para la evaluación específica de la psicopatía subclinica se ha desarrollado el Self-Report Psychopathy III (SRP-III) ${ }^{15}$ que contiene cuatro cuatro factores: manipulación interpersonal, insensibilidad afectiva, estilo de vida errático y tendencias criminales.

Complementariamente, cada vez es mayor la sensibilidad clínica ante la presencia de casos que, no son transgresores de las leyes ni caen en formas de criminalidad, pero que se denominan "psicopatas integrados" ${ }^{16}$, por ser verdaderos depredadores sociales; con éxito frecuente en su entorno, que no dudan en acciones que pueden ocasionar daños y perjuicios considerables a terceros o a grupos, y sin que ello les suponga ninguna emoción negativa.

Tabla 6. Trastorno Antisocial en el Trabajo:

Conducta aparente: Impresiona de valiente y/o temerario.

Conducta interpersonal: Beligerante, hipersensible a la crítica, provocador.

Estilo cognitivo: Implicado o incluso fanático. Patrones rígidos.

Percepción de sí mismo: Competitivo, dominante.

Mecanismo de defensa: Acting out.

En el caso del trastorno limite de personalidad, se presentan cambios bruscos y dramáticos en los objetivos, valores y aspiraciones profesionales. La inestabilidad de sus emociones y la intensidad de las mismas interfieren en su vida cotidiana. Los trastornos de identidad son frecuentes, no estando seguros de quiénes son en lo personal y en lo profesional, siendo difícil por ello dirigir su propia vida e intereses laborales.

El malestar significativo que perciben genera, en ocasiones, una desadaptación social y ocupacional, con importantes dificultades en la incorporación (el trastorno se suele desencadenar en la adolescencia o primera juventud, etapa de preparación profesional) y/o mantenimiento en el mundo laboral (por inadaptación, falta de organización y absentismo laboral) ${ }^{17}$. Presentan elevada vulnerabilidad a la opinión y rechazo de jefes y compañeros. El patrón lábil e inestable emocionalmente que presentan, les hace especialmente vulnerables a la presión ambiental y al estrés, utilizando como mecanismo de defensa la regresión. La imposibilidad de afrontar los estresores como adulto, puede empujar a la persona a retraerse a estadíos de desarrollo más tempranos, con una disminución del control de impulsos, ensoñaciones sobre deseos propios y dependencia de otra persona.

Cuando la situación clínica es estable, no existe consumo de drogas y hay un deseo manifiesto de integrarse en el mundo laboral, el papel del supervisor será especialmente importante estableciendo claros límites en las tareas, conductas y desempeño esperado, así como habilidad para manejar posibles discusiones e incluso enfrentamientos personales ${ }^{18}$. 
Tabla 7. Trastorno Límite en el Trabajo:

Conducta aparente: Impulsivo, caótico, desorganizado

Conducta interpersonal: Vulnerabilidad a la opinión ajena, comportamiento impredecible, manipulativo

Estilo cognitivo: Patrón de frecuentes cambios.

Percepción de sí mismo: Inquieto, conflictivo.

Mecanismo de defensa: Regresión.

\section{b) Abordaje psiquiátrico y psicológico (Grupo B)}

Un gran número de autores aseguran que vivimos en una sociedad que refuerza algunas conductas histriónicas y se muestran pesimistas respecto a la posibilidad de modificar los patrones básicos de este tipo de personalidad (por ejemplo Turkat) ${ }^{19}$. Este autor propone que en algunos casos, el entrenamiento en empatía puede ser útil para ayudar a la persona a despegarse de su punto de vista sobre el mundo. Horowitz ${ }^{20}$ plantea una integración de aspectos dinámicos y cognitivo-conductuales para la intervención en 4 fases:

1. Clarificación de la sintomatología, establecimiento de la alianza terapéutica, estabilización emocional y trabajo sobre patrones interpersonales desadaptativos.

2. Afrontamiento de cambios en el estado mental. Trabajo sobre aspectos como pensar antes de actuar, expresión clara y tranquila de ideas y emociones, evitación de la inundación emocional... como formas adaptativas de conseguir la atención.

3. Identificación y manejo de procesos defensivos de control. Se contraataca la tendencia a evitar temas conflictivos y se refuerza la observación de la conducta ajena.

4. Identificación y modificación de creencias irracionales y contradicciones en los esquemas sobre si mismo y los demás.

En el caso de la personalidad narcisista, se trata de un trastorno crónico y difícil de tratar ya que sus propias características obstaculizan la terapia y suponen un reto para el psicoterapeuta. Para la comprensión del caso será importante conocer las experiencias evolutivas que puedan relacionarse con el desarrollo del trastorno en edades tempranas, Millon y Everly ${ }^{2}$ señalan factores a considerar tales como la indulgencia parental y sobrevaloración, conductas explotadoras aprendidas y estatus de hijo único. Por lo general, las estrategias interventivas se centran en la identificación y análisis del patrón desadaptativo en términos de esquemas, creencias disfuncionales de grandiosidad, estilos y déficits de habilidades; en un segundo momento, el proceso terapéutico continúa con el cuestionamiento de ese patrón y la búsqueda de su sustitución por otro más adaptativo ${ }^{21}$.

Los sujetos con un trastorno antisocial de la personalidad raramente acuden a tratamiento y cuando es así, el porcentaje de eficacia es limitado. La falta de empatía, la incapacidad para establecer relaciones fiables, el desprecio por las normas sociales y, en los casos más graves, la conducta psicopática, impiden la alianza terapéutica, la capacidad de reflexión sobre el pasado y sobre las consecuencias futuras de la conducta. Cuando es posible la intervención, dos aspectos serán claves durante la psicoterapia: la identificación, manejo y autorregulación de la ira y el control de impulsos18. Respecto a su tratamiento farmacológico, estos dos factores pueden responder a sales de litio, carbamazepina o ácido valproico (en el caso de la hipomania e impulsividad) y a antipsicóticos, antidepresivos serotoninérgicos y benzodiacepinas (para la irritabilidad y episodios de cólera).

En cuanto al trastorno límite de personalidad, supone uno de los retos más difíciles en salud mental y que frecuentemente conlleva conductas autodestructivas y complicaciones médicas y sociales durante el tratamiento. Existe consenso general en que un objetivo básico será contener la impulsividad y regular las emociones. Una de las intervenciones sobre las que existe evidencia científica es la Terapia Dialéctica Conductual de Linehan ${ }^{22,23}$ que pretende la mejora de la eficacia interpersonal, la regulación emocional, 
el incremento de la tolerancia al malestar y la frustración e incrementar el autocontrol. Algunos de los fármacos más utilizados en este trastorno son ${ }^{24}$ : anti-psicóticos, empleados para tratar las cogniciones alteradas, ideas de referencia, disociaciones y alucinaciones; antidepresivos, prescritos principalmente para reducir la disforia asociada al rechazo personal; estabilizantes del estado de ánimo y anticonvulsivos, para regular los intensos cambios de humor, minimizando los estados de impulsividad y agresión; y benzodiacepinas, dirigidas a la reducción de la ansiedad, episodios de hostilidad y las alteraciones del sueño.

\section{c) Caso clínico. Consulta por adicciones en el lugar de trabajo en un trabajador con trastorno límite de la personalidad}

Auxiliar de enfermería de 30 años con adicción a benzodiacepinas que sustrae del lugar de trabajo y por lo que por la que ha solicitado voluntariamente un cambio de puesto a un servicio donde no tenga acceso a las mismas "las busco donde sea". Consumo alcohol (7-8 cervezas/día). Con anterioridad ha pasado por diversos tratamientos psiquiátricos y psicológicos que siempre ha abandonado. Antecedentes de depresión en padre y madre.

Autoconcepto y descripción de sí misma: "Tengo sentimientos de vacío, necesidad de cambiar cosas para que cambien cosas... me gustaría ser más asertiva y enfrentarme a las situaciones... quiero volverme mala, me importan demasiado los demás, me siento sola... Soy un bicho raro"

Tras el proceso de evaluación y diagnóstico se considera el caso como un trastorno límite de la personalidad con importantes rasgos dependientes y trastorno de ansiedad, siendo la adicción un efecto secundario a todo ello. La intervención se centró en el sentido de sí misma, el autoconcepto y déficit de autoestima (más allá de la descripción de sí misma que obtenía de la observación de su sintomatología), importancia de la autoeficacia profesional (pasando de la I.T. a la reincorporación laboral); definición y búsqueda de un espacio personal, y trabajo sobre la dependencia afectiva de los otros (madre, pareja, compañeros de trabajo...), autocontrol de la polaridad inhibición/ agresividad y aprendizaje de comunicación asertiva (aplicándola en la vida personal y laboral), control de impulsos, elaboración de trauma (abusos). Con apoyo farmacológico se procedió a la retirada progresiva de las benzodiacepinas, esto junto al trabajo psicoterapéutico comentado, proporcionó a la paciente los recursos necesarios para la deshabituación y reincorporación laboral.

\section{GRUPO C (Ansiosos o temerosos)}

Este grupo se caracteriza por un patrón penetrante de temores anormales, incluyendo relaciones sociales, separación y necesidad de control. Incluye el trastorno de la personalidad dependiente, por evitación y obsesivo-compulsivo.

El trastorno de la personalidad por DEPENDENCIA expresa una necesidad general y excesiva de que se ocupen de uno, lo que ocasiona un comportamiento sumiso, de adhesión y temores de separación, que empieza al inicio de la edad adulta y se da en varios contextos, como lo indican cinco (o más) de los siguientes ítems:

1. Tiene dificultades para tomar las decisiones cotidianas si no cuenta con el consejo y reafirmación por parte de los demás.

2. Necesidad de que otros asuman la responsabilidad en las principales parcelas de su vida.

3. Tiene dificultades para expresar el desacuerdo con los demás debido al temor a la pérdida de apoyo o aprobación. 
4. Tiene dificultades para iniciar proyectos o para hacer las cosas a su manera (debido a la falta de confianza en su propio juicio o en sus capacidades más que a una falta de motivación o de energía)

5. Va demasiado lejos llevado por su deseo de lograr protección y apoyo de los demás, hasta el punto de presentarse voluntario para realizar tareas desagradables.

6. Se siente incómodo o desamparado cuando está solo debido a sus temores exagerados a ser incapaz de cuidar de sí mismo.

7. Cuando termina una relación importante, busca urgentemente otra relación que le proporcione el cuidado y el apoyo que necesita.

8. Está preocupado de forma no realista por el miedo a que le abandonen y tenga que cuidar de sí mismo.

El trastorno de la personalidad por EVITACIÓN plantea un patrón general de inhibición social, sentimientos de inferioridad e hipersensibilidad a la evaluación negativa, que comienzan al principio de la edad adulta y se dan en diversos contextos, como lo indican cuatro (o más) de los siguientes ítems:

1. Evita trabajos o actividades que impliquen un contacto interpersonal importante debido al miedo a las críticas, la desaprobación o el rechazo.

2. Es reacio a implicarse con la gente si no está seguro de que va a agradar.

3. Demuestra represión en las relaciones íntimas debido al miedo a ser avergonzado o ridiculizado.

4. Está preocupado por la posibilidad de ser criticado o rechazado en las situaciones sociales.

5. Está inhibido en las situaciones interpersonales nuevas a causa de sentimientos de inferioridad.

6. Se ve a sí mismo socialmente inepto, personalmente poco interesante o inferior a los demás.

7. Es extremadamente reacio a correr riesgos personales o a implicarse en nuevas actividades debido a que pueden ser comprometedoras.

El trastorno OBSESIVO-COMPULSIVO de personalidad se expresa mediante un patrón general de preocupación por el orden, el perfeccionismo y el control mental e interpersonal, a expensas de la flexibilidad, la espontaneidad y la eficiencia, que empieza al principio de la edad adulta y se da en diversos contextos, como lo indican cuatro (o más) de los siguientes ítems:

1. Preocupación por los detalles, las normas, las listas, el orden, la organización o los horarios, hasta el punto de perder de vista el objeto principal de la actividad.

2. Perfeccionismo que interfiere con la finalización de las tareas (p. ej., es incapaz de acabar un proyecto porque no cumple sus propias exigencias, que son demasiado estrictas).

3. Dedicación excesiva al trabajo y a la productividad con exclusión de las actividades de ocio y las amistades (no atribuible a necesidades económicas evidentes).

4. Excesiva terquedad, escrupulosidad e inflexibilidad en temas de moral, ética o valores (no atribuible a la identificación con la cultura o la religión).

5. Incapacidad para tirar los objetos gastados o inútiles, incluso cuando no tienen un valor sentimental.

6. Es reacio a delegar tareas o trabajo en otros, a no ser que éstos se sometan exactamente a su manera de hacer las cosas.

7. Adopta un estilo avaro en los gastos para él y para los demás; el dinero se considera algo que hay que acumular con vistas a catástrofes futuras.

8. Muestra rigidez y obstinación. 


\section{a) Características y manejo en el lugar de trabajo (Grupo C)}

En el ámbito laboral, los trastornos de este grupo pueden suponer una dificultad para la toma de decisiones.

Cuando existe un trastorno de personalidad dependiente, la persona evita iniciar nuevos trabajos y tareas fuera de la rutina normal o que conlleven un esfuerzo considerable. Simplemente pensar en empleos que aumenten su carga habitual de trabajo puede provocar en ellos debilidad, ansiedad y un sentimiento general de agotamiento ${ }^{20}$.

Son personas que requieren excesivamente a los demás para su validación, aunque tienen dificultades para establecer vínculos saludables $\mathrm{e}$ independientes con los compañeros de trabajo y con frecuencia les falta confianza en sí mismos y seguridad, por lo que se pueden mostrar como sumisos. Estas características favorecen que prefieran trabajos en los que tienen que realizar tareas para otras personas, en los que puedan evitar responsabilidades y toma de decisiones. Con frecuencia se auto-evalúan como ineptos, confían más en las capacidades ajenas que en las propias, amplifican sus fallos y presentan elevado temor a equivocarse, por lo que buscan con frecuencia la confirmación, consejo y ayuda de compañeros y/o superior. La frecuente ayuda que reciben, les refuerza y confirma su idea de ineptitud, por lo que el círculo se cierra. Solo con actitudes de confianza, pruebas de realidad y seguimiento en las tareas (no ayuda directa o evitación de las mismas) por parte de sus compañeros y supervisores, se conseguirá incrementar el sentido de auto-eficacia de estas personas.

Por estas características, para evitar conflictos en las relaciones, la persona dependiente tiende a la introyección como mecanismo de defensa. Es decir, más allá de identificarse o depender de los otros, internaliza a los demás para crear una unión interpersonal estable, aunque eso suponga perder la identidad propia y la autonomía.

Tabla 8. Trastorno Dependiente en el Trabajo:

Conducta aparente: Incompetente, desamparado, vulnerable.

Conducta interpersonal: Sumisión, dependencia

Estilo cognitivo: Crédulo, ingenuo.

Percepción de sí mismo: Inepto, inadecuado.

Mecanismo de defensa: Introyección.

Igual que ocurre en personas con trastorno de personalidad por dependencia, cuando predomina una personalidad evitativa existe una alta hipersensibilidad al rechazo. En este caso, además, se sienten perturbadas por su propio aislamiento social, su retraimiento y su incapacidad de mantener vínculos interpersonales estrechos. Para gratificar las necesidades y anhelos que no pueden ser realizados en la realidad, pueden recurrir a la fantasía como un instrumento de obtener un medio seguro en el que mostrar afectos, ira, u otras emociones que de otra manera no verían su expresión.

Se muestran especialmente alertas en el lugar de trabajo en la relación con compañeros como forma de anticiparse al rechazo. Su aislamiento provoca la evitación de posibles momentos de socialización laboral (la pausa para el café, momentos de descanso, etc.).

El estilo cognitivo indeciso provoca dificultad para la toma de decisiones laborales lo que retroalimenta su baja autoestima y autoeficacia dentro del grupo de trabajo y exige una energía psíquica que les hace mostrarse frecuentemente como crónicamente tensos y fatigados. En este sentido, una adecuada definición de funciones y objetivos del puesto de trabajo puede funcionar como una guía de actuación que facilite la tarea al trabajador con un trastorno de la personalidad por evitación.

Tabla 9. Trastorno Evitativo en el Trabajo:

Conducta aparente: Cauteloso, alerta, tímido.

Conducta interpersonal: Aislamiento socio-profesional.

Estilo cognitivo: Distraído, indeciso.

Percepción de sí mismo: Rechazado.

Mecanismo de defensa: Fantasía. 
En el trastorno de la personalidad obsesivo-compulsiva, se observa a un trabajador rígido en sus esquemas mentales, inflexible ante el cambio y que puede sentirse molesto si la rutina se ve alterada debido a su obsesión por el orden. Por consiguiente, son personas ansiosas $\mathrm{y}$, en ocasiones, con dificultad para completar las tareas y tomar decisiones al no poder realizar la actividad laboral con el perfeccionismo auto-exigido.

La consecuencia de un pensamiento obsesivo (por ejemplo "me voy a equivocar en el diagnóstico de este paciente") es siempre la ansiedad. El trabajador necesita eliminar tanto la ansiedad como la consecuencia prevista de ese pensamiento ("se va a morir por mi culpa”). En ocasiones, para neutralizar la obsesión, la persona realiza una compulsión, una acción repetitiva que puede ser mental (por ejemplo rezar, contar...) o motora (pisar las baldosas de determinada manera, lavarse las manos...). También puede realizar comprobaciones (del trabajo realizado un número no adaptativo de veces, si ha apagado la llave de la luz, el gas...), conductas de seguridad (como preguntar a alguien cercano), evitación (de sitios, circunstancias, personas, etc. que facilitan que se desencadene la obsesión), de vigilancia y razonamiento... el problema, es que estas conductas no hacen más que exacerbar las obsesiones. En los casos más graves, la intensidad de las obsesiones y lo disruptivo de las compulsiones pueden llegar a impedir la correcta integración en un entorno laboral y el propio proceso de realización de una tarea. Es por ello importante, que cuando se observe dicha sintomatología, se anime al trabajador a pedir ayuda especializada, antes de que la situación se cronifique.

Las personas que tienen personalidad obsesivo-compulsiva suelen sentirse incómodas y ansiosas en situaciones que están fuera de su control y como consecuencia de ello pueden tener dificultad para mantener relaciones interpersonales positivas y sanas. Pueden tener una actitud diferente con compañeros o subordinados que con supervisores, con tendencia más autoritaria que igualitaria. Para mantener la conformidad y aceptabilidad social, pueden utilizar la formación reactiva como mecanismo de defensa y control de impulsos.

Sin embargo, su tenacidad y meticulosidad puede ser reforzada en no pocas ocasiones en la organización como un trabajador que sigue criterios de "excelencia". Hecho que no hace más que agravar la sintomatología.

Tabla 10. Trastorno Obsesivo en el Trabajo:

Conducta aparente: Disciplinado, perfeccionista, tenaz, meticuloso en su trabajo.

Conducta interpersonal: Respetuoso, recto, normativo.

Estilo cognitivo: Esquemas mentales rígidos, inflexibilidad ante el cambio.

Percepción de sí mismo: Escrupuloso, ordenado, auto-exigente.

Mecanismo de defensa: Formación reactiva.

\section{b) Abordaje psiquiátrico y psicológico (Grupo C)}

Diversos autores ${ }^{7,13,25}$ proponen que es esencial comenzar por áreas deficitarias y comportamientos desadaptativos, como la falta de habilidades para dar prioridades, para manejar el tiempo, para solucionar problemas y el temor a cometer errores. Para ello recomiendan trabajar mediante técnicas cognitivo-conductuales aspectos tales como la eficacia, la gestión del tiempo (de trabajo y de ocio, de desconexión), programación de actividades, entrenamiento en relajación, en autoinstrucciones, detención de pensamientos rumiativos y modificación de suposiciones subyacentes. Esta labor se enfrentará no obstante a procesos de resistencia y de rigidez mental, ya que la inflexibilidad de pensamiento y/o de conducta es común en estas personas, causando ello, en los casos más graves, déficit cognitivos de funciones ejecutivas que regulan el procesamiento de la información y las estrategias adaptativas.

Por otra parte, comprender el trastorno en la historia de vida propia, implicará analizar su etiología en función de aspectos tales como la posible sobreprotección y control parental, el comportamiento compulsivo aprendido, el aprendizaje de responsabilidades y la valoración personal en función de logros (más que por lo que uno es). 
Con respecto al tratamiento farmacológico del trastorno obsesivo compulsivo de la personalidad, no existen estudios controlados que evalúen su eficacia ${ }^{13}$. Si la sintomatología dificulta en gran medida la vida cotidiana y/o laboral, como ocurre en algunos casos de trastorno obsesivo-compulsivo, el tratamiento recomendado incluye fármacos que actúen sobre el sistema serotoninérgico, tales como la fluvoxamina (50-300 mg/día), otros inhibidores de la recaptación de la serotonina (ISRS) a dosis equivalentes o clomipramina (25-225 mg/día), siendo la respuesta al tratamiento mejor cuando existe comorbilidad con el Eje I como los trastornos de ansiedad y/o del estado de ánimo.

\section{c) Caso clínico. El último en salir del Centro de Salud, un caso de trastorno obsesivo de personalidad}

Médico de Pediatra de 50 años. Plantea que en ocasiones supera las tres horas de retaso en consulta, desde su organización le hicieron cambios de horario, siendo el profesional con menor cupo pero se mantienen los problemas. Acude a su trabajo antes de la hora para intentar planificarse y adelantar trabajo pero siempre es el último en salir, con conflictos por ello con compañeros por la hora de cierre del centro. Según sus propias palabras: “...me cabreo si voy a toda pastilla y si voy lento también. No soporto dejar algo a la mitad, gestiono mal el tiempo, voy contra-reloj y se me han gastado las pilas... Cada día llegar al trabajo es como ir a un examen del que siempre salgo con un -2, así todos los días de mi vida, ir a trabajar no es eso. Cada vez tengo más cosas que hacer además de ver al paciente (burocracia, papeleos...) y no tengo tiempo, no sé cómo lo hacen mis compañeros..." "Me siento raro, extraño, plano, pesimista, con ira contenida, tengo mal manejo emocional, creo que no me pregunto por las emociones ni propias ni ajenas". "Tengo nula tolerancia a la frustración, me escandalizo pensando que me gustaría tener otra vida..." "Desde niño siempre he sido así, yo era feliz con un microscopio, tenía amigos y jugaba con ellos... pero me daban un poco igual..."

Más allá de una carga de trabajo real y objetiva en el puesto de trabajo, la evaluación clínica confirma la existencia de un trastorno obsesivo compulsivo de personalidad en comorbilidad con un trastorno de ansiedad que dificulta la gestión del tiempo, la tolerancia ante las dificultades, el abordaje de cada consulta de pediatría, la diferenciación entre lo importante y lo urgente y la flexibilidad cognitiva en la toma de decisiones.

El abordaje clínico requirió de una baja laboral, tratamiento farmacológico de la sintomatología y proceso psicoterápico que está en curso.

\section{OTROS TIPOS DE ALTERACIONES DE LA PERSONALIDAD}

\section{Trastorno de Personalidad NO ESPECIFICADO}

El trastorno no especificado se caracteriza porque los síntomas del paciente no encajan con los criterios de algún trastorno específico. Un ejemplo de esto es lo que se denomina el trastorno de personalidad mixta, donde los síntomas que se manifiestan en conjunto, producen un malestar clínicamente significativo o bien el deterioro de una o más áreas de la actividad del sujeto. Algunos ejemplos que se incluyen dentro de esta categoría son:

- Trastorno de la personalidad negativa: Caracterizado por una agresión pasiva, en la cual, la persona adopta una actitud negativa a resistirse a las exigencias y expectativas rutinarias.

- Trastorno depresivo de la personalidad: Comprende elevada autocrítica, desánimo, actitud crítica hacia los otros de manera mantenida y tendencia a sentirse culpable.

Además, es importante señalar que pueden existir ALTERACIONES Y CAMBIOS DE PERSONALIDAD DESPUÉS DE UN DAÑO CEREBRAL. En este sentido, en un marco de salud laboral, tras un accidente de trabajo o enfermedad médica con daño 
cerebral, y ante la sospecha o comunicación de compañeros y/o familiares del trabajador, sería necesario evaluar:

1. Si existe una alteración persistente de la personalidad que representa un cambio del patrón característico de personalidad previo del individuo.

2. Existen evidencias a partir de la historia, el examen físico o los hallazgos de laboratorio de que la alteración es una consecuencia fisiológica directa de una enfermedad médica.

3. La alteración no se explica mejor a partir de otro trastorno mental

4. La alteración no ocurre exclusivamente durante el transcurso de un delirium y no cumple criterios de demencia.

5. La alteración es causa de malestar clínico significativo o deterioro social, ocupacional o en otras importantes áreas de funcionamiento.

6. Especificar tipo:

- Tipo lábil: Si la característica predominante es la labilidad afectiva.

- Tipo desinhibido: Si la característica predominante es un pobre control de impulsos como por ejemplo, indiscreciones sexuales, etc.

- Tipo agresivo: Si la característica predominante es la conducta agresiva.

- Tipo apático: Si la característica predominante es una marcada apatía e indiferencia.

- Tipo paranoide: Si la característica predominante es la suspicacia o la ideación paranoide.

- Otro Tipo: Si la característica predominante no es ninguna de las citadas, por ejemplo: cambio de personalidad asociada a epilepsia.

- Tipo combinado: Si predominan más de una de las características en el cuadro clínico.

\section{- Tipo sin especificar.}

En los traumatismos craneoencefálicos graves, la prevalencia de cambios y trastornos de personalidad se sitúa entre el $40-60 \%$ de los casos. En la clínica, dichos cambios postraumáticos más frecuentes son: apatía y pérdida de interés por el entorno, embotamiento afectivo, irritabilidad, episodios de conducta explosiva, problemas de impulsividad, desinhibición y euforia, conducta egocéntrica e infantil, suspicacia, etc. En general se observan dos principales grupos de síntomas: relacionados con déficits en el control y modulación emocional y los que se encuentran vinculados con un descenso de la capacidad para mantener una conducta orientada a un fin. El primero genera mayor estrés en los allegados y rechazo social, pero el segundo, si predomina, resulta de peor pronóstico, siendo un reto su abordaje clínico y socio-laboral ${ }^{26}$.

Más allá de la clasificación diagnóstica del DSM-IV existen otros tipos de personalidad (no trastornos propiamente dichos) que creemos importante señalar brevemente en esta revisión por su importante relación con el mundo laboral y la importancia de su consideración en los procesos de vigilancia de la salud. Se trata de categorizaciones de la personalidad en "tipos" según patrones de conducta y de los aspectos positivos de la personalidad.

\section{PERSONALIDAD Y PATRONES DE CONDUCTA}

En 1957 Rosenman et al ${ }^{27}$ (cardiólogos del Hospital Monte Sinaí en San Francisco), describieron un estilo de comportamiento que llamaron patrón de conducta Tipo A, que constituye un factor de riesgo para la cardiopatía isquémica (se trata de un factor de riesgo que opera al margen de otros factores de riesgo como el tabaco, la hipertensión y la obesidad). Tienen un patrón de rápida activación, mantenimiento conductual de la misma y lenta recuperación. Estas personas tienen 2,5 veces más probabilidades de presentar 
angina de pecho o infarto de miocardio. El patrón de conducta tipo A ha sido definido como un componente acción-reacción con gran vulnerabilidad al estrés integrado por ${ }^{28}$ :

- Componentes formales: habla rápida, tensión en la musculatura facial, excesiva actividad psicomotora y otros manierismos.

- Actitudes y emociones: hostilidad, impaciencia, ira y agresividad.

- Aspectos motivacionales: motivación de logro, competitividad y ambición.

- Conductas abiertas o manifiestas: urgencias de tiempo, implicación en el trabajo.

- Aspectos cognitivos: necesidad de control ambiental y estilo atribucional característico

Las personas que muestran este tipo de comportamiento son llamados individuos Tipo-A y en oposición a ellos se encuentra el individuo Tipo B, referido a un estilo de vida más relajado, no apresurado y maduro.

En el trabajo, el patrón de conducta tipo A facilita gran implicación laboral, sobretodo en relación con el rendimiento y los resultados finales, pero con poca capacidad de disfrute de la actividad y del proceso de desarrollo de la misma. Asumen el descanso o el ocio como pérdida de tiempo. Con tendencia a la actividad permanente, su vida gira en torno al trabajo, al logro y al éxito, dan la impresión de no tener nunca suficiente. Dado que para ellos su valía personal depende de sus logros y cualquier muestra de debilidad puede ser fatal, suelen tener un gran temor al fracaso, pues lo consideran un indicativo de su falta de valor personal. Para los trabajadores con personalidad tipo A, el sentido del trabajo lo proporciona el posible éxito en el mismo, la posición y el prestigio obtenido, el sueldo, la variedad de la tarea, la posibilidad de control y la motivación de logro ${ }^{29}$. Sin embargo, la exigencia personal, la dificultad de delegar, la competitividad y la irritabilidad ante la frustración pueden ocasionarles una gran desmotivación, conflicto e insatisfacción ${ }^{30}$.

Por otro lado, en la última década de los 90 y principios de los años 2000, se ha descrito un tipo de personalidad relacionada con un alto riesgo de trastornos cardiovasculares. Denollet y colaboradores ${ }^{31-34}$ han sugerido que la personalidad Tipo D (de distress) se caracteriza por una afectividad negativa (tendencia a la preocupación y visión negativa de los acontecimientos, acompañada a menudo de sentimientos de tristeza, irritabilidad, tensión crónica y bajo nivel de bienestar subjetivo) e inhibición social (tendencia al distanciamiento de los demás, inhibición de la expresión emocional y bajos niveles de apoyo social percibido). Estos autores señalan que este estilo de personalidad supone un afrontamiento del estrés en el que existe un sesgo atencional hacia los estímulos negativos y predominan respuestas evitativas ante los mismos, con el resultado de una mayor sintomatología somática.

El estrés negativo (distress) propio de la personalidad Tipo D, puede provocar la enfermedad cardiovascular de dos formas:

- Directa: El estrés al que está expuesto el sujeto influye en los mecanismos fisiológicos que determinan la enfermedad cardiovascular.

- Indirecta: El estrés influye en comportamientos de mal autocuidado que incrementan la gravedad de la enfermedad cardiovascular

En un reciente artículo de revisión sobre este tema ${ }^{35}$, los autores señalan el impacto negativo de la personalidad Tipo D sobre la salud mental (con mayor sintomatología depresiva, ansiedad, estrés postraumático, estilos pasivos de afrontamiento y bajo apoyo social), sobre la salud física (somatizaciones y trastornos cardiovasculares) y problemas en el lugar de trabajo, en comparación con trabajadores con otros estilos de personalidad:

- Tienen mayores índices de absentismo ${ }^{36}$

- Reportan mayores índices de agotamiento (vital exhaustion) ${ }^{37}$ 
- Perciben su trabajo como más estresante y tienen mayores índices de desgaste profesional (burnout) ${ }^{38}$

- Tienen mayor riesgo de padecer estrés pos-traumatico ante agresiones físicas en el lugar de trabajo ${ }^{39}$

Algunos autores ${ }^{40}$ hablan también de un patrón de conducta Tipo F (en alusión a la f de "femenino" y de "fibromialgia"), para describir un conjunto de rasgos obsesivos, perfeccionistas y auto-exigentes que se expresan como forma de regular la autoestima en entornos donde existe falta de feedback, de retroalimentación sobre el desempeño de la tarea de forma contingente con el esfuerzo realizado para llevarla a cabo. Este patrón se caracterizaría además por una tendencia a la complacencia hacia los demás, evitación de los conflictos interpersonales y manejo inadecuado de la agresividad, predominando en mujeres que padecen síndromes dolorosos crónicos tales como la fibromialgia.

\section{ASPECTOS SANOS DE LA PERSONALIDAD}

A pesar de que el presente trabajo se refiere a trastornos y alteraciones de la personalidad, no quisiéramos terminar este artículo sin hacer una alusión a los aspectos sanos de la personalidad y su relación con el ámbito laboral.

Uno de los grandes objetivos de la psicología del trabajo actual es no sólo detectar los indicadores que causan problemas de salud, sino también aquellos que proporcionan lugares saludables para el trabajo. Respondiendo a estos planteamientos se ha desarrollado una Psicología Positiva del Trabajo que trata de enfatizar los aspectos de la organización del trabajo que favorecen tanto el desarrollo de empresas saludables como de personas sanas y positivas ${ }^{41}$. En relación con ello, se ha insistido en aquellos aspectos de la personalidad que favorecen el desarrollo organizacional, la convivencia grupal, la productividad y la innovación empresarial. En este sentido dos son los modelos principales que se han presentado como aspectos de la personalidad que favorecen la resistencia a los efectos del estrés y al desarrollo de una buena salud mental adaptativa y proactiva.

Históricamente el primero de ellos ha sido el concepto de Personalidad Resistente que supone que las variables de "compromiso", "control" y "reto" son factores que favorecen la fortaleza en el trabajo y la resistencia al estrés. Otro modelo más reciente es el de Capital Psicológico, que insiste en que las variables positivas de la persona son un capital psicológico real que favorece la regulación interna de las organizaciones y la capacidad personal para el desarrollo de una óptima salud mental. Las variables que se proponen como integrantes del capital psicológico son las de "optimismo", "control", "autoeficacia" y "resiliencia".

\section{CONCLUSIÓN}

Los rasgos de personalidad explican una proporción significativa de la varianza de resultados laborales tales como la productividad, satisfacción laboral o absentismo, según un modelo interactivo entre variables individuales (capacidades, creencias, rasgos de personalidad, valores), situacionales (cultura corporativa, normas grupales) y variables relacionadas con la actividad laboral (tareas específicas) ${ }^{42}$.

Las personas con trastornos de personalidad ven afectadas la capacidad de funcionar de un modo autónomo y competente y conseguir las propias metas, así como la posibilidad de ajustarse de manera eficiente y flexible al medio socio-laboral. En consecuencia, el trabajador con un trastorno de personalidad presentará alteraciones en la forma de percibir los acontecimientos externos, en la manera de interpretarse a si mismo y a los demás, en los afectos, en las relaciones interpersonales y en el control de los impulsos. Con gran probabilidad, serán percibidos por sus compañeros como manipuladores, molestos, buscadores de atención, conflictivos, etc. Una herramienta útil en la evaluación 
del nivel general de disfunción de las relaciones interpersonales en posibles trastornos de personalidad es el Inventory of Interpersonal Problems (IIP) ${ }^{43}$ y sus subescalas para trastornos de personalidad: sensibilidad interpersonal, ambivalencia interpersonal, agresividad, necesidad de aprobación social y carencia de habilidades sociales.

La importancia de la detección temprana e intervención será esencial, tanto para el propio trabajador como para su entorno, clima laboral y funcionamiento de la organización; así como para la sociedad en general, ya que los trastornos mentales son, en España, la segunda causa de baja laboral y el tercer grupo de enfermedades que genera más gasto al sistema sanitario público ${ }^{44}$. En ocasiones, la posibilidad de detección de estos trastornos está más allá de los recursos habituales en los Servicios de Prevención y por ello es necesaria la estrecha colaboración con Servicios de Salud Mental y programas de atención al empleado. A pesar de eso, los Servicios de Salud Laboral y Prevención de Riesgos Laborales tendrán un papel fundamental, ya que a través de la detección de conflictos laborales e intervención sobre los mismos, tienen un lugar privilegiado de diagnóstico clínico, siendo para ello esencial la dotación adecuada en personal de estos servicios (especialmente Técnicos Superiores en Factores Psicosociales y Médicos del Trabajo), así como la formación en salud mental de los equipos.

Desde el punto de vista de la intervención, sólo con la colaboración entre las diferentes entidades implicadas: Direcciones y Gerencias de las Organizaciones, Departamentos de Recursos Humanos, Servicios de Prevención y Servicios de Salud Mental (Centros de Salud Mental, de Rehabilitación Laboral, etc.), y con una perspectiva multidisciplinar, podremos trabajar en la detección, ayuda e integración sociolaboral de las personas con un trastorno de personalidad.

\section{BIBLIOGRAFÍA}

1. Moreno Jiménez, B. Psicología de la personalidad. Procesos. Madrid: Thomson.

2. Millon T y Everly GS. Personality and its disorders. Nueva York: Wiley (1985).

3. Mingote J.C.; del Pino P.; Huidobro A.; Gutiérrez D.; de Miguel I.; Gálvez M. El paciente que padece un trastorno psicótico en el trabajo: diagnóstico y tratamiento. Med Segur Trab 2007; 52 (208): 29-52.

4. Mingote J.C.; Gálvez M; del Pino P.; Gutiérrez D. El paciente que padece un trastorno depresivo en el trabajo. Med Segur Trab 2009; 55(214): 41-63.

5. DSM-IV

6. Bernstein D.P., Useda D., Siever L.J. Paranoid personality disorder: Review of the literature and recommendations for DSM-IV. Journal of Personality Disorders 1993; 7: 56-62.

7. Beck A.T. y Freeman A. Cognitive therapy of personality disorders. New York: Guilford Press (1990)

8. Gabbard G.O. Subtypes of narcissistic personality disorder. Bull Menninger Clin 1989; 53:527-532

9. Shapiro, D. Neurotic styles. New York: Basic Books. 1965

10. Oldham, JM y Morris LB. Autorretrato de la personalidad. Gerona: Tikal (1995)

11. Hirigoyen M.F. El acoso moral en el trabajo. Madrid: Paidós (2001).

12. Graña J.L., Muñóz J.J. y Navas E. Características psicopatológicas, motivacionales y de personalidad en drogodependientes en tratamiento de la Comunidad de Madrid. Madrid: Agencia Antidroga (2007).

13. Caballo V.E. Manual de trastornos de la personalidad. Descripción, evaluación y tratamiento. Madrid: Síntesis (2004).

14. Jakobwitz, S. y Egan, V. The dark triad and normal personality traits. Personality and Individual Differences 2006; 40(2): 331-339.

15. Paulhus, D.L., Hemphill, J.D. y Hare, R.D. (en prensa). Manual for the Self-Report Psychopathy Scale. Toronto: Multi-Health Systems.

16. Pozueco Romero, J.M.. Psicopatas integrados. Madrid: Editorial EOS (2010)

17. Castán N. Inserción laboral de personas con trastorno límite de personalidad (TLP). Cuad Psiquiatr Comunitaria 2005; 5(2): 165-177. 
18. Unterberg M.P. Personality: Personalities, Personal Style and Trouble Getting Along. En: J.P. Kahn y A.M. Langlieb. Mental Health and Productivity in the Workplace. San Francisco, CA: Jossey-Bass (2003).

19. Turkat I.D. The personality disorders: A psychological approach to clinical management. Nueva York: Pergamon (1990).

20. Horowitz M.J. Histrionic personality disorder. En G.O. Gabbard (dir.), Treatments of psychiatric disorders ( $2^{\mathrm{a}}$ ed.). Washington: American Psychiatric Press (1995).

21. Sperry L. Cognitive behaviour therapy of DSM-IV personality disorders. Philadelphia, PA: Bruner \& Mazel (1999).

22. Linehan MM, Armstrong HE, Suárez A, Allmon D y Heard HL. Cognitive-behavioral treatment of chronically parasuicidal borderline patients. Archives of General Psychiatry 1991; 48:1060-1064.

23. Linehan MM. Skills training manual for treating borderline personality disorder. New York: Guilford (1993). (Trad. al castellano, Paidós 2003).

24. Dimeff LA, Mc David J, Linehan MM. Pharmacotherapy for borderline personality disorder: a review of the literature and recommendations for treatment. Journal of Clinical Psychology in Medical Settings 1999; 6:113-138.

25. Freeman A, Pretzer J, Fleming B, Simon KM. Clinical applications of cognitive therapy. Nueva York: Plenum (1990).

26. Muñóz JM, Paúl M, Pelegrín C, Tirapu J. Factores de pronóstico en los traumatismos craneoencefálicos Revista de Neurología 2001; 32(4): 351-364.

27. Rosenman RH, Friedman M, Straus R, Wurm M, Kositchek R, Hahn W, et al. A predictive study of coronary heart disease. JAMA 1964; 189: 15-22.

28. Friedman M y Rosenman, R. (1974). Type-A behaviour and your heart. N.Y.: Knopf.

29. García MA, Berrios MP. El significado del trabajo en personas con patrón de conducta tipo A. Psicothema 1999; 11(2): 357-366.

30. Sorensen G, Jacobs DR, Pirie P, y Folsom A. Relationships among type A behaviour, employment experiences and gender: The Minnesota Heart Survey. Journal of Behavioral Medicine 1987; 10(4): 323-336.

31. Denollet J., Brutsaert D. Personality, disease severity and the risk of long-term cardiac events in patients with a deseased ejection fraction after myocardial infarction. Circulation 1998; 97: 167-173.

32. Denollet J. Type D personality: A potencial risk factor refined. Journal of Psychosomatic Research 2000; 49(4): 255-266.

33. Denollet J y Van Heck G. Psychological risk factors in heart disease. What Type D personality is (not) about. Journal of Psychosomatic Research 2001; 51: 465-468.

34. Pedersen S y Denollet J. Type D personality, cardiac events and impaired quality of life: a review. European Journal of Cardiovascular Prevention and Rehabilitation 2003; 10(4): 241-248.

35. Mols F y Denollet J. Type D personality in the general population: a systematic review of health status, mechanisms of disease and work-related problems. Health and Quality of Life Outcomes 2010. 8 (9).

36. Hanebuth D, Meinel M y Fischer JE. Health-related quality of life, psichosocial work conditions and absenteeism in an industrial same of blue- and white-collar employees: a comaparison of potential predictors. J Occup Envion Med 2006; 48(1): 28-37.

37. Preckel D, von Kanel R, Kudielka BM, Fischer JE. Overcommitment to work is associated with vital exhaustion. Int Arch Occup Environ Health 2005; 78(2): 117-122.

38. Oginska-Bulik N. Occupational stress and its consequences in healthcare professionals: the role of type D personality. Int j Occup Med Environ Health 2006; 19(2): 113-122.

39. Kunts MJJ, Bogaerts S, Winkel FW. Peer and inmate aggression, Type D personality and post-traumatic stress among Dutch prison workers. Stress and Health 2009; 25(5): 387-395.

40. Mingote JC. En Alvarez Romero M, García Villamisar, D. El Síndrome del Perfecionista: el Anancástico. Sevilla: Ed. Almuzara (2007).

41. Alez Linley P, Harringtopn S, Garcea N. Oxford Handbook of Positive Psychology at Work. Oxford: Oxford University Press (2010).

42. Furnham, A. (2001). Psicología organizacional. El comportamiento del individuo en las organizaciones. México: Oxford University Press.

43. Horowitz LM, Rosenberg SE, Baer BA, Ureño G, Villaseñor VS. Inventory of Interpersonal Problems: Psichometric properties and clinical applications. Journal of Consulting and Clinical Psychology 1988, 56: 885-892.

44. Moreno JO, López-Bastida J, Montejo-González AL, Osuna-Guerrero R y Duque-González B. The socioeconomic cost of mental iones in Spain. European Journal of Health Economics 2009; 10(4): 361-369.

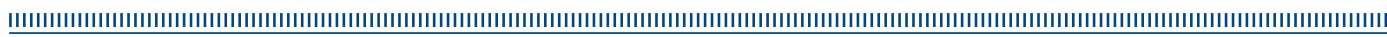

